\title{
PERSONALIDAD Y METAS DE RENDIMIENTO EN ESCOLARES DE PRIMARIA
}

\author{
Benito León del Barco \\ Departamento de Psicología y Antropología \\ Universidad de Extremadura \\ bleon@unex.es \\ Santiago Mendo Lázaro \\ Departamento de Psicología y Antropología \\ Universidad de Extremadura \\ smendo@unex.es \\ María Isabel Polo del Río \\ Departamento de Psicología y Antropología \\ Universidad de Extremadura \\ mabelpdrio@unex.es \\ Fernando Fajardo Bullón \\ Departamento de Psicología y Antropología \\ Universidad de Extremadura \\ fernandofajardo@unex.es \\ Margarita Gozalo Delgado \\ Departamento de Psicología y Antropología \\ Universidad de Extremadura \\ mgozalo@unex.es
}

Fecha de Recepción: 9 Abril 2019

Fecha de Admisión: 30 Abril 2019

\section{RESUMEN}

Existe una gran mayoría de estudios que relacionan las metas con factores de aprendizaje, incluido el rendimiento. Otro grupo de investigaciones buscan cómo determinados factores personales determinan un tipo de meta. Con relación a los factores personales, autores como Harackiewicz, Barron, Tauer, Carter y Elliot (2000) sostienen que las metas que mantienen los alumnos dependen de variables personales, al considerar éstas como constructos estables y de orden disposicional. Como objetivo, nos hemos planteado estudiar las relaciones entre las metas de rendimiento (metas de ejecución, o metas centradas en el yo) y los diferentes rasgos de personalidad en escolares de primaria. En este trabajo de investigación participan 550 alumnos de $5^{\circ}$ y $6^{\circ}$ de Educación Primaria. La edad media fue de 10.73 años (SD= .632; rango 10-12); 47.5\% mujeres y el $52.5 \%$ de varones. Se han utilizado el factor "Metas de Rendimiento" del Cuestionario de Metas Académicas, CMA (Hayamizu y Weiner, 1991) y el Cuestionario de personalidad BFQ-NA (Del Barrio, 
Carrasco y Holgado, 2006). El ANOVA muestra que los sujetos con altas metas de rendimiento, logran puntuaciones mayores $(p<0.005)$ que aquellos con baja en todos los factores a excepción del factor inestabilidad emocional, en el que son los sujetos con bajas metas de rendimiento los que obtienen puntuaciones $(p=0.037)$ mayores. Los resultados obtenidos muestran una clara asociación entre las metas de rendimiento de los niños de primaria y los diferentes factores de la personalidad. De este modo aquellos niños con elevadas metas de rendimiento, su personalidad se caracterizaría por una mayor autonomía, perseverancia, compromiso, creatividad, entusiasmo, autoconfianza, sociabilidad, entusiasmo o prosociabilidad y un menor neuroticismo y ansiedad. Estos resultados coinciden parcialmente con otras investigaciones que encuentran correlaciones entre las metas de rendimiento y la conciencia (Chen y Zhang, 2011; McCabe, Van Yperen, Elliot y Verbraak, 2013).

Palabras clave: metas académicas; metas de rendimiento; personalidad; alumnos; educación primaria

\section{ABSTRACT}

Personality and performance goals in primary education students. There are a large majority of studies that relate goals to learning factors, including performance. Another group of research seeks how certain personal factors determine a type of goal. With regard to personal factors, authors such as Harackiewicz, Barron, Tauer, Carter and Elliot (2000) argue that the goals that students maintain depend on personal variables, considering them as stable and dispositional constructs. As an objective, we have considered studying the relationship between performance goals (performance goals, or goals centered on the self) and the different personality traits in primary education students. This research involved 550 students of 5 th and 6th grade of Primary Education. The mean age was 10.73 years (SD =.632, range $10-12$ ); $47.5 \%$ female and $52.5 \%$ male. The "Performance Goals" factor of the Achievement Goal Questionnaire (Hayamizu \& Weiner, 1991) and the BFQ-NA Personality Questionnaire (Del Barrio, Carrasco, \& Holgado, 2006) have been used. The ANOVA shows that subjects with high performance goals achieve higher scores $(p<0.005)$ than those with low scores in all factors except for the emotional instability factor, in which subjects with low performance goals obtain scores $(p=0.037)$ greater. The results obtained show a clear association between the performance goals of primary education students and the different personality factors. In this way, those children with high performance goals, their personality would be characterized by greater autonomy, perseverance, commitment, creativity, enthusiasm, self-confidence, sociability, enthusiasm or prosociability and less neuroticism and anxiety. These results coincide partially with other studies that find correlations between performance goals and awareness (Chen \& Zhang, 2011; McCabe, Van Yperen, Elliot, \& Verbraak, 2013).

Keywords: academic goals; performance goals; personality; students; primary education

\section{INTRODUCCIÓN}

En el estudio de los diferentes tipos de metas académicas se ha considerado tradicionalmente las metas de aprendizaje y las metas de rendimiento. Los estudiantes en clase se implican en una tarea y se esfuerzan por dos tipos de razones. Por un lado, quieren adquirir conocimientos, aumentar su competencia, dominar y disfrutar con la tarea, en definitiva, quieren aprender y mejorar sus capacidades, estamos hablando de metas de aprendizaje, también denominadas metas de dominio y metas centradas en la tarea (Ames, 1992; Dweck, 1986; Nicholls y Miller, 1984). Por otro lado, hay estudiantes que se esfuerzan porque quieren sacar buenas notas, avanzar en los estudios, quieren parecer listos ante los demás y evitar que les perciban como incompetentes, en definitiva quieren demostrar su valía y capacidad y buscan valoraciones positivas de su desempeño, nos referimos a 
metas de rendimiento, descritas, también como metas de ejecución o metas centradas en el yo (Ames, 1992; Dweck, 1986; Nicholls y Miller, 1984).

De este enfoque bidimensional, pasando por uno tridimensional (Elliot y Harackiewicz, 1996) y un modelo 2x2 (Elliot, 2005; Elliot y McGregor, 2001), hemos llegado a un modelo 3x2 (Elliot, Murayama y Pekrun, 2011). El modelo tridimensional (Elliot y Harackiewicz, 1996) considera dentro de las metas de rendimiento la diferencia entre metas de aproximación y de evitación (dirección de la meta). El modelo 2x2 (Elliot, 2005; Elliot y McGregor, 2001), añade la diferencia entre metas de aproximación y de evitación a las metas de aprendizaje (orientación de meta). Por último, el modelo 3x2 (Elliot, Murayama y Pekrun, 2011) pretende aumentar la precisión del modelo 2x2 considerando varios referentes como la tarea (task), el yo (self) y el otro (other) en la evaluación de las competencias de los estudiantes.

\section{Personalidad y metas académicas}

No es fácil clasificar las investigaciones en torno a las metas académicas. Existe una gran mayoría de estudios que relacionan las metas con factores de aprendizaje, incluido el rendimiento. Otro grupo de investigaciones buscan cómo determinados factores personales determinan un tipo de meta. Con relación a los factores personales, autores como Harackiewicz, Barron, Tauer, Carter y Elliot, (2000) sostienen que las metas que mantienen los alumnos dependen de variables personales, al considerar éstas como constructos estables y de orden disposicional. Se han estudiado variables como la percepción que tienen los alumnos sobre la inteligencia. Aquellos alumnos que creen que la inteligencia depende del esfuerzo se inclinan más por las metas de aprendizaje frente a los que consideran la inteligencia como un rasgo estable que asumen en mayor medida metas de rendimiento.

Otros autores han estudiado la relación entre la conducta agresiva y las metas (Wentzel y Asher, 1995; Little y Garber, 2004). También, se han examinado las relaciones entre los diferentes tipos sociométricos (populares-preferidos, rechazados-agresivos, rechazados-tímidos e ignorados-olvidados) y la conducta prosocial con las metas académicas en muestras de adolescentes de Educación Secundaria (Inglés, Martínez-González, Valle, García-Fernández y Ruiz-Esteban, 2011; Inglés, Aparisi, García-Fernández, Núñez y Martínez, 2016).

\section{Nuestro estudio}

Se han analizado las relaciones entre las metas del modelo 2x2 (Elliot y McGregor, 2001) con los factores del modelo de los cinco grandes de personalidad, este modelo es una teoría central en el estudio de los rasgos de la personalidad (Allport, 1937). McCabe, Van Yperen, Elliot y Verbraak (2013) encuentran en una muestra de estudiantes universitarios relaciones fuertes ente los factores conciencia y amabilidad con las metas de aproximación de aprendizaje. Asimismo, el factor neuroticismo se relaciona positivamente con las metas de evitación. En esta misma línea de trabajo con el modelo de los cinco grandes de personalidad y el modelo 2x2, Chen y Zhang (2011) encuentran que la personalidad es mejor predictor que el temperamento.

Nos hemos planteado estudiar las relaciones entre las metas de rendimiento (metas de ejecución, metas centradas en el yo) y los diferentes rasgos de personalidad en escolares de primaria.

\section{MÉTODO}

\section{Participantes}

La muestra de participantes estuvo constituida por 550 alumnos. La edad media fue de 10.73 años $(S D=.632$; rango $10-12) ; 47.5 \%$ mujeres y el $52.5 \%$ de varones. El alumnado cursaba $5^{\circ}$ 
(45.10\%) y 6은 (54.90\%) de Educación Primaria. La selección de los alumnos se realizó mediante un muestreo polietápico por conglomerados y selección aleatoria de los grupos en los centros que disponían de varias líneas en los cursos $5^{\circ}$ y $6^{0}$ de Primaria. El muestreo por conglomerados se llevó a cabo seleccionando al azar cuatro centros educativos públicos y concertados de Cáceres.

\section{Instrumentos}

Cuestionario de Metas Académicas (CMA). El cuestionario es una adaptación elaborado a partir del Cuestionario de Metas (Hayamizu, Ito y Yohiazaki, 1989; Hayamizu y Weiner, 1991) y traducido al castellano. La adaptación se ha llevado a cabo teniendo en cuenta el análisis factorial realizado por González, Torregrosa y Navas (2002) eliminando los ítems que saturaban menos para cada uno de los tres factores, de 20 ítems se han dejado 15. Por otro lado hemos añadido a estos 15 ítems (cinco por cada factor), 5 ítems que conforman otro factor nuevo extraído de la teoría planteada por Alonso (1991). El cuestionario, por tanto, está compuesto por 20 ítems que plantean razones por las que estudia el alumno. La escala de respuesta es de tipo Likert con 5 grados de frecuencia ( $1=$ nunca, $2=$ pocas veces, $3=$ algunas veces, $4=$ a menudo y $5=$ siempre).

Para este trabajo se ha utilizado como variable el factor "Metas de Rendimiento". Este factor explica el 8,5\% de la varianza y se refiere a las metas relacionadas con el "yo", la motivación de rendimiento y el miedo al fracaso, ejemplo: "Estudio porque deseo alcanzar una buena posición social en el futuro". Presenta un alfa de Cronbach de .824.

Cuestionario de personalidad BFQ-NA (Big Five Questionnaire - Niños y Adolescentes) creado por (Barbaranelli, Caprara y Rabasca, 1998 y traducido al castellano por Del Barrio, Carrasco y Holgado (2006a). Es una prueba que mide los cinco grandes factores de personalidad (Extraversión, Neuroticismo, Afabilidad, Apertura y Tesón) en población no adulta. El cuestionario consta de 65 ítems con una escala de respuesta tipo Likert de cinco intervalos (desde 1= "casi nunca" hasta 5= "casi siempre").

En España, Del Barrio, Carrasco y Holgado (2006b) estudiaron las características psicométricas del BFQ-NA con una muestra de 852 niños entre 8 y 15 años. En este estudio se obtuvo una buena fiabilidad del cuestionario (alfa de cronbach entre 0,78 y 0,88 ; test-retest entre 0,62 y 0,84 ). A su vez, el análisis factorial confirmatorio y exploratorio informaron de una estructura de cinco factores correspondientes con los cinco grandes (Soto et al, 2011).

\section{Procedimiento}

Los datos se obtuvieron a partir de cuatro escuelas públicas. Los consejos escolares de todos Ios centros acordaron participar. Seguimos las directrices éticas de la American Psychological Association (APA, 2009) y se solicitaron formularios de consentimiento informado a los padres y alumnos. No se les ofreció compensación, siendo totalmente voluntaria su participación. Los instrumentos se completaron ante un entrevistador entrenado y en el contexto del aula.

\section{RESULTADOS}

En primer lugar, se transforma la variable cuantitativa "Metas de Rendimiento" en una variable cualitativa mediante un criterio de percentiles, (33\%), asumiendo que el tercio, inferior, medio y superior de la muestra corresponden a bajas, medias y altas Metas de Rendimiento respectivamente. Posteriormente, con el objetivo de comprobar si existen diferencias en la personalidad en función de los diferentes grupos de metas de rendimiento, se lleva a cabo un ANOVA (Tabla 1). 
Tabla 1. Resultados de la prueba ANOVA. Factor Metas de Rendimiento (Bajas, Medias, Altas).

\begin{tabular}{|c|c|c|c|c|c|}
\hline & $\begin{array}{c}\text { Metas } \\
\text { Rendimiento }\end{array}$ & M & DT & $F$ & $p$ \\
\hline \multirow{3}{*}{$\begin{array}{c}\text { Factor conciencia } \\
\text { cuestionario BFQ-NA }\end{array}$} & Bajas & 48,71 & 9,37 & \multirow{3}{*}{30,309} & \multirow{3}{*}{, 000} \\
\hline & Medias & 55,89 & 6,25 & & \\
\hline & Altas & 58,13 & 8,67 & & \\
\hline \multirow{3}{*}{$\begin{array}{c}\text { Factor amabilidad } \\
\text { cuestionario BFQ-NA }\end{array}$} & Bajas & 48,77 & 7,92 & \multirow{3}{*}{14,834} & \multirow{3}{*}{, 000} \\
\hline & Medias & 53,56 & 8,88 & & \\
\hline & Altas & 55,18 & 5,43 & & \\
\hline \multirow{3}{*}{$\begin{array}{c}\text { Factor inestabilidad } \\
\text { cuestionario BFQ-NA }\end{array}$} & Bajas & 29,34 & 8,96 & \multirow{3}{*}{3,327} & \multirow{3}{*}{,037 } \\
\hline & Medias & 28,17 & 8,47 & & \\
\hline & Altas & 25,96 & 7,21 & & \\
\hline \multirow{3}{*}{$\begin{array}{l}\text { Factor extraversión } \\
\text { cuestionario BFQ-NA }\end{array}$} & Bajas & 41,10 & 6,91 & \multirow{3}{*}{6,512} & \multirow{3}{*}{,002 } \\
\hline & Medias & 43,15 & 5,27 & & \\
\hline & Altas & 44,37 & 5,09 & & \\
\hline \multirow{3}{*}{$\begin{array}{c}\text { Factor apertura } \\
\text { cuestionario BFQ-NA }\end{array}$} & Bajas & 31,47 & 6,44 & \multirow{3}{*}{16,414} & \multirow{3}{*}{, 000} \\
\hline & Medias & 35,10 & 5,30 & & \\
\hline & Altas & 36,73 & 5,84 & & \\
\hline
\end{tabular}

EI ANOVA muestra la existencia de diferencias significativas $(p<0.005)$ en los factores conciencia, amabilidad, inestabilidad, extraversión y apertura.

La comparación por pares de Bomferroni (Tabla 2), muestra que los sujetos con altas metas de rendimiento, logran puntuaciones mayores $(p<0.005)$ que aquellos con baja en todos los factores a excepción del factor inestabilidad emocional, en el que son los sujetos con bajas metas de rendimiento los que obtienen puntuaciones $(p=0.037)$ mayores.

Tabla 2. Resultados de la prueba post hoc Bonferroni.

\begin{tabular}{|c|c|c|c|c|c|}
\hline \multicolumn{6}{|c|}{ Bonferroni } \\
\hline Variable dependiente & $\begin{array}{l}\text { (I) Percentile } \\
\text { Metas de } \\
\text { rendimiento }\end{array}$ & $\begin{array}{l}\text { (J) Percentile } \\
\text { Metas de } \\
\text { rendimiento }\end{array}$ & $\begin{array}{l}\text { Diferencia de } \\
\text { medias (I-J) }\end{array}$ & ET & $p$ \\
\hline \multirow{2}{*}{$\begin{array}{c}\text { Factor conciencia } \\
\text { cuestionario BFQ- } \\
\text { NA }\end{array}$} & \multirow[b]{2}{*}{ Bajas } & Medias & $-7,18166^{*}$ & 1,19748 &, 000 \\
\hline & & Altas & $-9,42902^{*}$ & 1,26960 &, 000 \\
\hline \multirow{2}{*}{$\begin{array}{c}\text { Factor amabilidad } \\
\text { cuestionario BFQ- } \\
\text { NA }\end{array}$} & \multirow[b]{2}{*}{ Bajas } & Medias & $-4,79259^{*}$ & 1,15950 &, 000 \\
\hline & & Altas & $-6,41098^{*}$ & 1,22996 &, 000 \\
\hline \multirow{2}{*}{$\begin{array}{c}\text { Factor inestabilidad } \\
\text { cuestionario BFQ- } \\
\text { NA }\end{array}$} & \multirow[b]{2}{*}{ Bajas } & Medias & 1,17136 & 1,27351 & 1,000 \\
\hline & & Altas & $3,37553^{*}$ & 1,34345 &, 038 \\
\hline \multirow{2}{*}{$\begin{array}{c}\text { Factor extraversión } \\
\text { cuestionario BFQ- } \\
\text { NA }\end{array}$} & \multirow[b]{2}{*}{ Bajas } & Medias & $-2,04564$ &, 85866 & ,054 \\
\hline & & Altas & $-3,26647^{*}$ & ,91451 &, 001 \\
\hline \multirow{2}{*}{$\begin{array}{c}\text { Factor apertura } \\
\text { cuestionario BFQ- } \\
\text { NA }\end{array}$} & \multirow[b]{2}{*}{ Bajas } & Medias & $-3,62411^{*}$ & ,88607 &, 000 \\
\hline & & Altas & $-5,25203^{*}$ & ,93781 &, 000 \\
\hline
\end{tabular}




\section{DISCUSIÓN}

Se han analizado las relaciones entre las metas de rendimiento y los diferentes rasgos de personalidad en escolares de primaria. Los resultados obtenidos muestran una clara asociación entre las metas de rendimiento de los niños de primaria y los diferentes factores de la personalidad. De este modo aquellos niños con elevadas metas de rendimiento, su personalidad se caracterizaría una mayor autonomía, perseverancia, compromiso, creatividad, entusiasmo, autoconfianza, sociabilidad, entusiasmo o prosociabilidad y un menor neuroticismo, ansiedad o depresión.

La mayoría de estudios indican que la personalidad está fuertemente relacionada con las metas académicas. En este sentido, resultados hallados en un estudio con alumnado universitario muestran que la personalidad está fuertemente relacionada con la motivación académica, concretamente, las metas de rendimiento están relacionadas con un mayor equilibrio emocional y una alta responsabilidad, así como con alta extraversión, apertura a nuevas ideas y experiencias y también con más amabilidad, por parte del alumnado (Morán y Menezes, 2016). En la misma línea, otros estudios muestran que aquellos estudiantes con una alta conciencia, extraversión y franqueza, muestran altas metas de rendimiento (Payne, Youngcourt y Beaubien, 2007).

De forma específica, mientras que ciertos trabajos concluyen que las metas de rendimiento se han asociado positivamente con conciencia y extraversión, y negativamente con el neuroticismo (De Guzman et al., 2003; Heaven, 1990) estudios como los de Komarraju y Karau (2005) encontraron que dichas metas se explican mejor por la conciencia, el neuroticismo y la apertura, mientras que la conciencia explicaba la mayor variación. No obstante, con el modelo 2x2, Chen y Zhang (2011) en un estudio con 775 estudiantes, entre 12 y 19 años, encuentran una asociación positiva entre el neuroticismo y/o inestabilidad emocional con las metas de evitación del rendimiento. En este sentido, nuestros resultados en cuanto al factor neuroticismo se explicarían en base a una asociación entre las bajas metas de rendimiento con las metas de evitación del rendimiento. Habrá que seguir trabajando para aclarar estas cuestiones.

Por último, nuestro estudio contradice la visión estereotipada de los estudiantes que quieren ser los mejores, destacar y alcanzar el éxito. Tradicionalmente, estos comportamientos se han considerado desadaptativos (Hidi y Harackiewich, 2000).

Queremos indicar que este trabajo presenta varias limitaciones. El hecho de realizar un estudio transversal, impide establecer asociaciones de causalidad. Además, el uso exclusivo de los autoinformes y el uso de la percepción de los niños como única fuente de información y de recopilación de datos, obstaculiza la posibilidad de controlar el posible sesgo de los encuestados. Creemos que sería importante contar con otros informantes además de los involucrados directamente. Consideramos que los profesores se encuentran en una situación privilegiada para analizar las Metas Académicas que pueden evaluarlas en la situación diaria de clase. Por último, es importante destacar que este es un estudio que incluye solo algunas variables potenciales asociadas a las metas de rendimiento. Esto conlleva la falta de control de variables contextuales, como las diferentes características de la escuela o el aula.

\section{REFERENCIAS BIBLIOGRÁFICAS}

Allport, G. W. (1937). Personality: A psychological interpretation. New York: Holt.

Alonso, J. (1991). Motivación y aprendizaje en el aula. Cómo enseñar a pensar. Madrid: Santillana.

American Psychological Association (2009). Publication Manual of the American Psychological Association, Sixth Edition.

Ames, C. (1992). Classrooms: Goals, structures, and student motivation. Journal of Educational

Psychology, 84, 261-271. doi:10.1037/0022-0663.84.3.261 
Barbaranelli, C., Caprara, G. V., y Rabasca, A. (1998). Manuale del BFQ-C. Big Five Questionnaire Children. OS Organizazioni Speciali, Firenze.

Chen, C., y Zhang, L. (2011). Temperament, personality and achievement goals among Chinese ado-

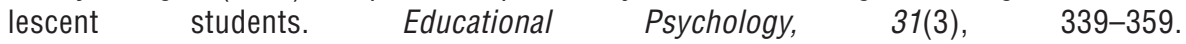
doi:10.1080/01443410.2011.559310

De Guzman, I. N., Calderon, A., y Cassaretto, M. (2003). Personality and academic

achievement in university students. Revista de Psicologia, 21(1), 119-143.

Del Barrio, M. V., Carrasco, M. A., y Holgado, P. (2006a). BFQ-NA cuestionario de los Cinco Grandes para niños y adolescentes (adaptación a la población española). Madrid: TEA.

Del Barrio, V., Carrasco, M. A., y Holgado, F. P. (2006b). Factor structure invariance in the Children's Big Five Questionnaire. European Journal of Psychological Assessment, 22(3), 158-167.

Dweck, C. S. (1986). Motivational process affects learning. American Psychologist, 41, 1040-1048. doi:10.1037/0003-066X.41.10.1040

Elliot, A. J. (2005). A conceptual history of the achievement goal construct. In A. Elliot \& C. Dweck (Eds.), Handbook of competence and motivation (pp. 52-72). New York, NY: Guilford P.

Elliot, A. J., y Harackiewicz, J. M. (1996). Approach and Avoidance Achievement Goals and Intrinsic Motivation: A Mediational Analysis. Journal of Personality and Social Psychology, 70(3), 461475. doi:10.1037/0022-3514.70.3.461

Elliot, A. J., y McGregor, H. A. (2001). A 2 × 2 achievement goal framework. Journal of Personality and Social Psychology, 80(3), 501-519. doi:10.1037//0022-3514.80.3.501

Elliot, A. J., K. Murayama y R. Pekrun. (2011). A 3 × 2 achievement goal model. Journal of Educational Psychology. 103(3). 632-648. doi:10.1037/a0023952

González, C., Torregrosa, G., y Navas, L. (2002). Analysis of the goals for school children in primary and secondary school. Revista Española de Orientación y Psicopedagogía, 13(1), 69-87. doi:10.5944/reop.vol.13.num.1.2002.11587

Harackiewicz, J. M., Barron, K. E., Tauer, J. M., Carter, S. M., y Elliot, A. J. (2000). Short-term and long-term consequences of achievement goals: Predicting interest and performance over time. Journal of Educational Psychology, 92, 316-330. doi:10.1037/0022-0663.92.2.316

Hayamizu, T., Ito, A., y Yohiazaki, K. (1989). Cognitive motivational process mediated by achievement goal tendencies. Japonese Research, 31, 179-189.

Hayamizu, T., y Weiner, B. (1991). Una prueba del modelo de Dweck de los objetivos de logro en relación con las percepciones de la capacidad. The Journal of Experimental Education, 59(3), 226-234.

Heaven, P. C. (1990). La actitud y la personalidad se correlacionan con la motivación de logro entre los estudiantes de secundaria. Personalidad y diferencias individuales, 11(7), 705-710.

Hidi, S. y Haracliewich, J. M. (2000). Motivating the academically unmotivated: A critical isue for the 21s century. Review of Educational Research, 70 (2), 151-179.

Inglés, C. J., Aparisi, D., García-Fernández, J. M., Núñez, J. C., y Martínez, M. C. (2016). Relationship between sociometric types and academic goals in a sample of Spanish students of Secondary Education. Universitas Psychologica, 15(1), 233-244. doi:10.1114/Javeriana.upsy15-1.rsma

Inglés, C. J., Martínez-González, A. E., Valle, A., García-Fernández, J. M., y Ruiz-Esteban, C. (2011). Prosocial behavior and academic motivation in Spanish High School Students. Universitas Psychologica, 10(2), 451-465.

Komarraju, M. y Karau, S. J. (2005). The relationship between the big five personality traits and academic motivation. Personality and individual differences, 39(3), 557-567.

Little, S., y Garber, J. (2004). Interpersonal and achievement orientations and specific stressors pre- 
dict depressive and aggressive symptoms. Journal of Adolescent Research, 19(1), 63-84.

McCabe, K. O., Van Yperen N. W., Elliot, A. J., y Verbraak, M. (2013). Big Five personality profiles of context-specific achievement goals. Journal of Research in Personality, 47, 698-707. doi:10.1016/j.jrp.2013.06.003

Morán, C. y Menezes, E. (2016). La motivación de logro como impulso creador de bienestar: su relación con los cinco grandes factores de la personalidad. International Journal of Developmental and Educational Psychology, 2(1), 31-40.

Nicholls, J. G., y Miller, A. T. (1984). Reasoning about the ability of self and others: A developmental study. ChildDevelopment. 55, 1990-1999. doi:10.2307/1129774

Payne, S. C., Youngcourt, S. S. y Beaubien, J. M. (2007). A meta-analytic examination of the goal orientation nomological net. Journal of applied psychology, 92(1), 128.

Soto, G., Ferrándiz, C., Sáinz, M., Ferrando, M., Prieto, M.D., Bermejo, R. y Hernández, D. (2011). Características psicométricas del cuestionario de personalidad BFQ-NA (Big Five Questionnaire - Niños y Adolescentes). Aula Abierta, 39 (1), 13-24.

Wentzel, K. R., y Asher, S. R. (1995). The academic lives of neglected, rejected, popular and controversial children. ChildDevelopment, 66, 3, 754-763.doi:10.2307/1131948 